\section{SEM Demonstrations for Younger Visitors}

Charles Lyman and Kathleen Repa

Lehigh University, Bethlehem, PA

Each year we show hundreds of students and visitors magnified images of common objects in our SEM. the best samples to use in SEM demonstrations for children are those which have detail that can just barely be seen with the naked eye or with a light microscope. Ideally, the sample would be something with which the child is already familiar, and one which can be explored at several different magnifications.

The simplest specimen is a penny attached to a stub by carbon or silver dag. Mount the coin such that the back side, showing the Lincoln Memorial, faces upward. As shown in Figure 1, the statue of Lincoln inside the memorial can be seen with the naked eye. To get the lowest possible magnification,
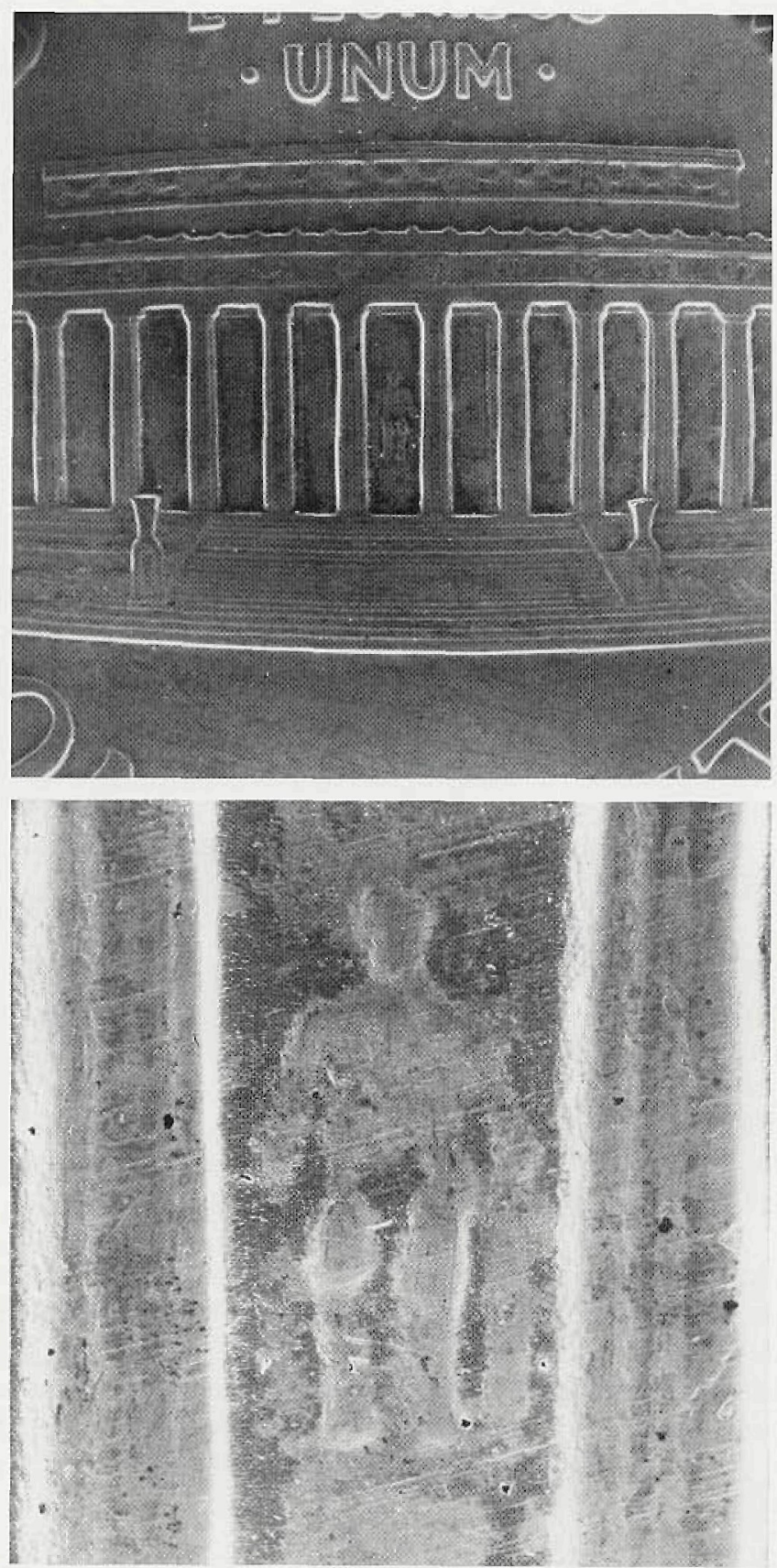

Figure 1: (Upper) Low magnification image of the reverse side of a penny showing Lincoin's statue between the columns. Working distance at maximum producing an original magnification of $12 x$. (Lower) Higher magnification of the Lincoln statue between two columns lower the specimen with the z-drive to provide the longest working distance. The EDS unit may be used to show that the copper penny is not pure copper but rather an alloy containing zinc in solid solution. The zinc makes the penny harder than if it were pure copper. The composition of the interior of the penny can be assessed by deeply scratching the penny and collecting an $x$-ray spectrum from within the scratch (see Figure 2)

Insects are fascinating to viewers of all ages, but they are particularly useful for getting and holding a child's attention. For example, it is very effective to begin a demonstration at a magnification which shows only the compound eye of a bumblebee and to ask the children to guess what they are looking at. The magnification can then be reduced to show the head and antennae looking straight out of the microscope monitor. The exploration continues by tilting and translating the bee inside the specimen chamber. The bee is well-adapted for the collection of pollen. Figure 3 shows two magnifications of the leg of a bee. Note the pollen particles that have been "collected" on the hairs protruding from the leg.

David Scharf is a recognized expert on insect photography with the SEM. Note the centerfold in the March 1995 issue of this publication. Scharf's technique is to image the insect uncoated at $5 \mathrm{kV}$ with a long working distance, a small final aperture, and at a low magnification. These settings provide a large depth-of-field. Just dag a leg onto a SEM stub. Beetles tend to survive the microscope vacuum better than other insects. To see some excellent examples, take a look at David Scharf's book entitled Magnifications: Photography with the Scanning Electron Microscope published by Schocken Books, New York, 1977.

If the children are to be given a "hands on" demonstration, a simpler approach can be taken to image previously expired insects. We have successfully mounted insects at Lehigh by dagging one end of a wire to a SEM stub and sticking the other end to the insect's body with a little dab of TorrSeal low-vapor pressure epoxy. This allows the legs to hang free and the insect can be manipulated to better imaging positions than when glued directly to the stub. To facilitate imaging at 20 or $30 \mathrm{kV}$, the insect may be coated with Au-Pd since the magnifications to be used are so low that the coating itself cannot be resolved.

A human hair coated with gold-palladium can also be interesting, especially since the typical diameter is $100 \mu \mathrm{m}=0.1 \mathrm{~mm}$, approximately the resolution limit of the unaided eye. It might be interesting to include a dog or cat hair on the same stub.

Filaments from new and blown light bulbs are also interesting, particularly when mounted on the same stub for comparison. If the bulb had a long life, the tungsten wire may be thinner in some areas due to evaporation of tungsten atoms onto the inner surface of the glass. Filaments with coils provide spectacular 3 dimensional stereo images. Again, for large depth-of-field use a long working distance and a small final aperture. Keep the left picture at zero tilt. tilt the right image about 7 degrees toward the Everhart-Thornley electron detector, translate the specimen to place the image feature in its original location, and refocus with the $z$-motion only. The two pictures should be oriented so that the tilt axis is parallel to the length of the viewer's nose. Sometimes it is useful to mark a

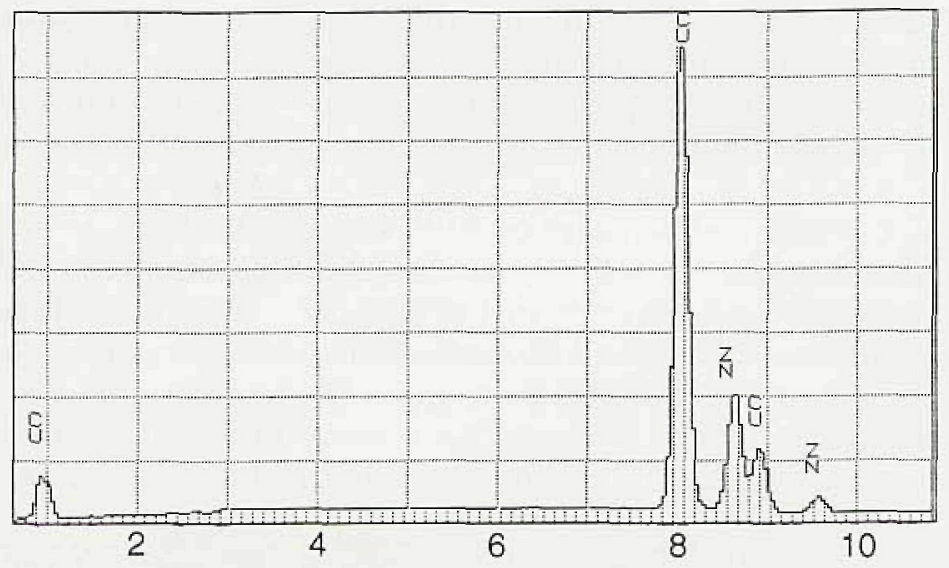

Figure 2. X-ray spectrum of penny from an area with a fresh deep scratch taken with a Be-window Si(Li) energy-dispersive spectrometer (EDS) Note that the penny is made of an alloy of copper and zinc which exhibits greater hardness than pure copper. 
particular specimen feature on the screen with a wax pencil so exactly the same area can be photographed in the second picture. One must be careful when breaking the glass envelope to avoid flying glass. Be sure to wear safety glasses and break the bulb only inside a safety cloth. Try not to damage the rather fragile filament.

In addition, any flat-polished metallurgical specimen can be examined in the SEM with great results. For example, compare the images of a flat-polished specimen of basalt (a mineral) from the reflecting light microscope with the image from a SEM in backscattered electron mode. In the backscattered electron image, the phases with the higher average atomic number are brighter. Use the EDS unit to confirm this by identifying the elements in the different phases.

But the greatest thrill is to invite your guests to move one of these specimens around by themselves. To explore on one's own with a microscope is a terrific way to stimulate scientific curiosity. Good luck - have a great day with your young visitors!
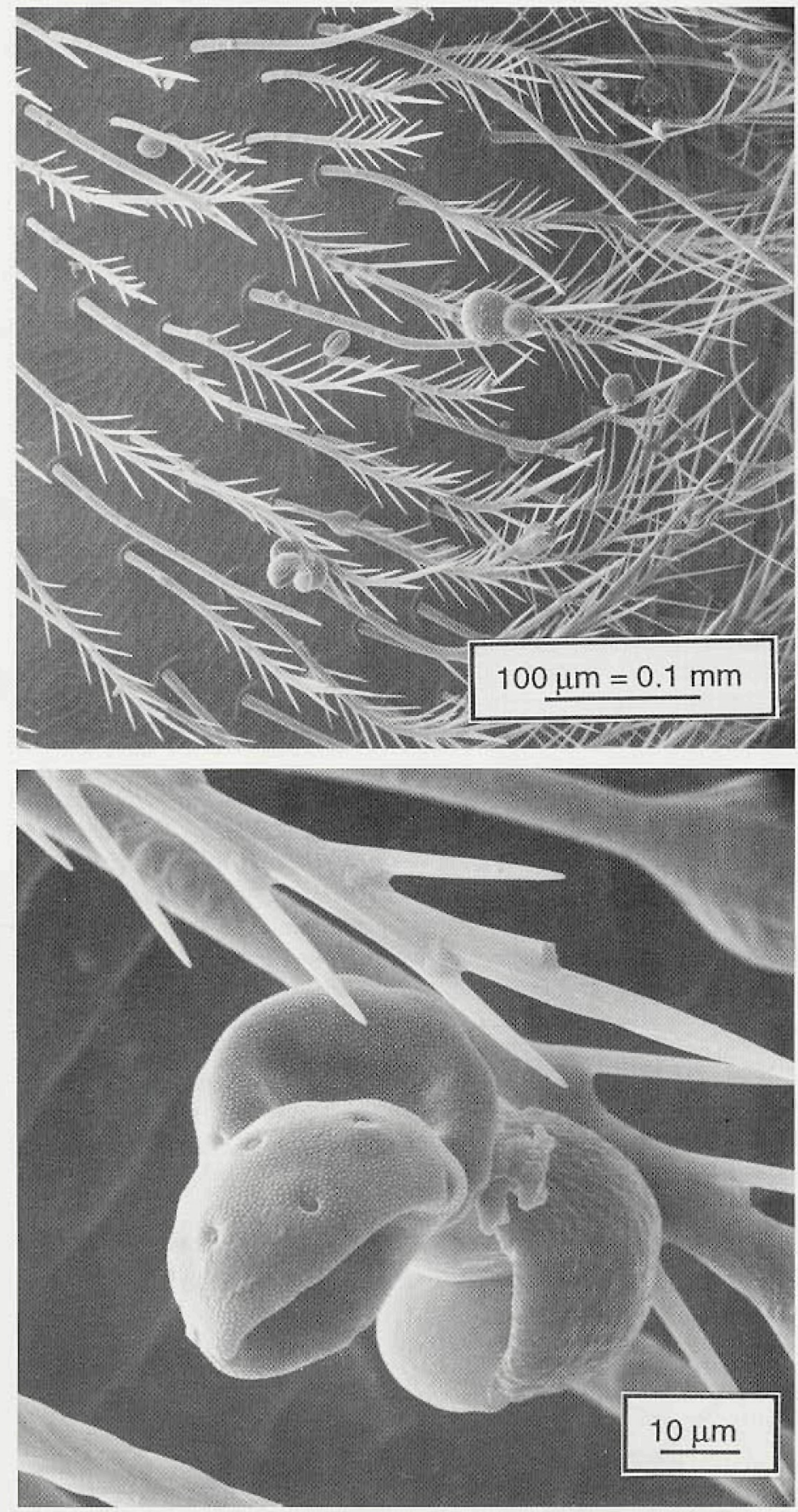

Figure 3: (Upper) Low magnification image of the hair on the leg of a bumblebee. (Lower) Higher magnification image showing pollen grains attached to the spiny structure of the

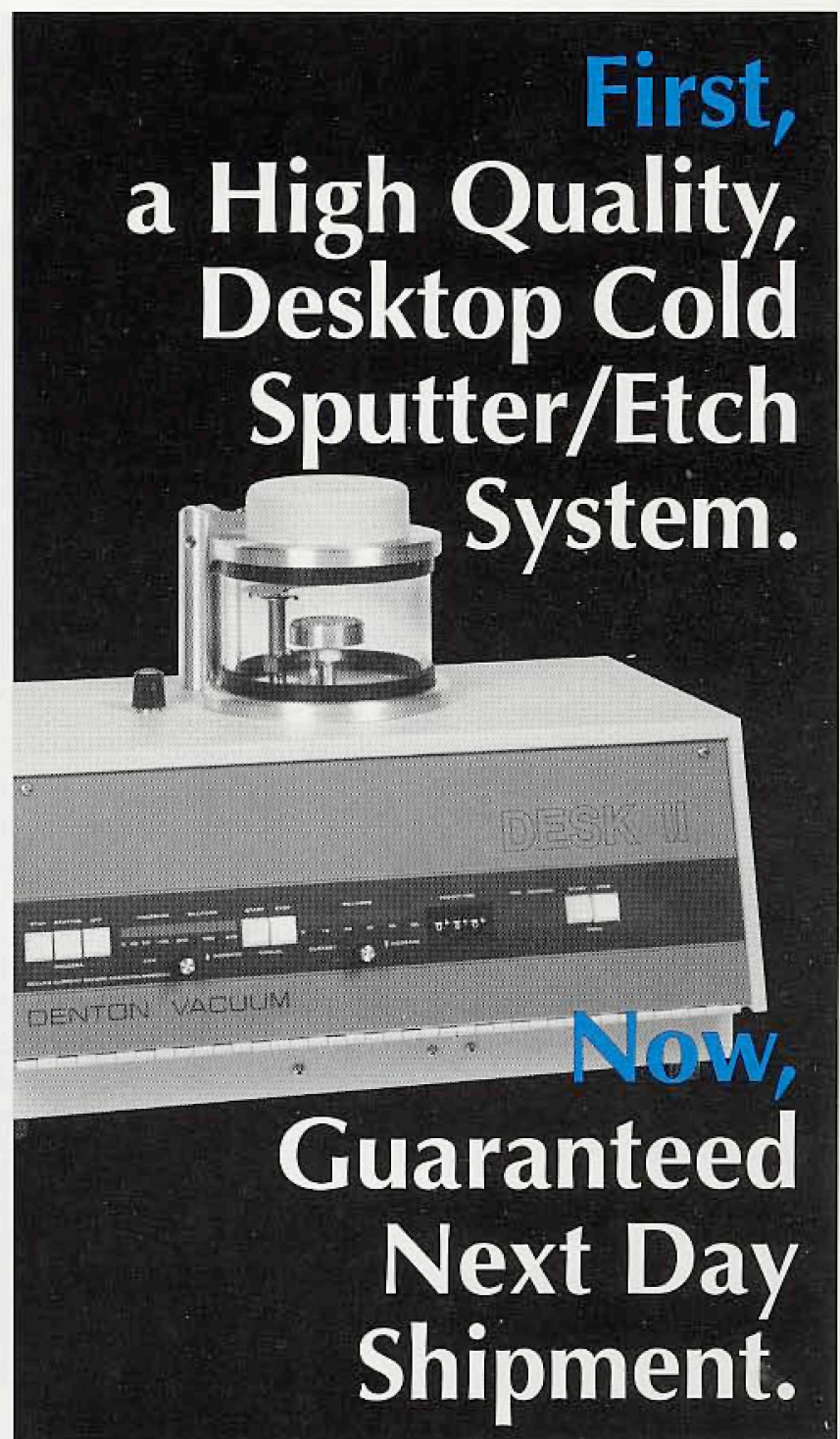

Order Denton's standard Desk II System by noon today and get guaranteed shipment tomorrow or Denton pays the freight.

The Desk II produces uniform, conductive, fine grain Au/AuPd coatings in under three minutes and is the highest quality desktop system available.

\section{The Desk II features:}

automatic or manual operation

Easy to read digital vacuum and current gauges

- Optional carbon evaporation accessory

For more information, look no further than Denton... where both Quality and Delivery invite comparison.

\section{DENTON $\begin{aligned} & 1259 \text { North Church St. } \\ & \text { Moorestown, NJ 08057 USA }\end{aligned}$ TACUUMM Tel: (609) 439-9100 INC.

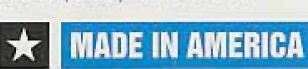

Next day shipment must be requested at time of order.

Decades of Experience In EM Specimen Prep Equipment 